\title{
Repenser la gestion des ravageurs du cotonnier en Afrique de l'Ouest
}

\author{
Thierry Brévault ${ }^{1,2, *}$, Djibril Badiane ${ }^{3}$, Régis Goebel ${ }^{2,4}$, Alain Renou ${ }^{2,4}$, Idrissa Téréta ${ }^{5}$ et \\ Pascal Clouvel ${ }^{2,4}$ \\ ${ }^{1}$ CIRAD, UPR AIDA, Centre commun ISRA-IRD, Dakar, Sénégal \\ 2 AIDA, Univ Montpellier, CIRAD, Montpellier, France \\ ${ }^{3}$ ISRA, CRA de Tambacounda, Tambacounda, Sénégal \\ ${ }^{4}$ CIRAD, UPR AIDA, 34398 Montpellier, France \\ 5 IER, CRRA de Sikasso, Sikasso, Mali
}

\begin{abstract}
Résumé - Les insectes ravageurs représentent une contrainte majeure à l'augmentation du rendement en culture cotonnière en Afrique de l'Ouest. Les filières cotonnières se sont engagées, à des degrés divers, dans des démarches de gestion intégrée des ravageurs pour réduire l'utilisation des insecticides et limiter en amont les risques sanitaires et environnementaux. Toutefois, un changement fondamental des pratiques, au bénéfice de la stimulation des services de régulation naturelle des ravageurs, s'impose pour mettre au point des systèmes de production encore plus économes en pesticides et plus résilients. Les leviers permettant de stimuler les processus écologiques de régulation naturelle des ravageurs sont multiples et redevables d'actions à différentes échelles, du champ cultivé au territoire, mais nécessitent d'approfondir notre connaissance du système de vie des ravageurs et de leurs ennemis naturels. Ils nécessitent aussi une plus grande prise en compte de la perception et des pratiques des acteurs dans une approche participative de la gestion collective des ressources et des processus d'innovation. À terme, il s'agit de construire une organisation territorialisée d'actions individuelles et collectives de gestion des ravageurs et des services écosystémiques, incluant des dimensions écologiques, économiques et sociales.
\end{abstract}

Mots clés : processus écologiques / paysage / agroécosystème / biodiversité

\begin{abstract}
Reconsidering cotton pest management in West Africa. Insect pests represent a major constraint to the increase of cotton yield in West Africa. The cotton value chain has been involved to varying degrees in integrated pest management approaches (IPM) to reduce the use of insecticides and to limit health and environmental risks. However, a fundamental change in farming practices, oriented toward the stimulation of natural pest control services, is needed to invent more pesticide-efficient and resilient production systems. The levers for promoting natural pest regulation are multiple and actionable at different scales from field to territory, but still require deepening our knowledge of the life system of pests and their natural enemies. They also necessitate to better take into account the perception and practices of stakeholders, in a participatory approach of the collective management of resources and processes of innovation. Ultimately, the aim is to build a territorialized organization of individual and collective actions for the management of pests and ecosystem services, including ecological, economic and social dimensions.
\end{abstract}

Keywords: ecological processes / landscape / agroecosystem / biodiversity

\section{Introduction}

Les insectes ravageurs représentent une contrainte majeure à l'augmentation des rendements en production cotonnière en

\footnotetext{
*Auteur de correspondance $:$ thierry.brevault@cirad.fr
}

Afrique de l'Ouest, avec des pertes de récolte de l'ordre de $25 \%$ en moyenne malgré les traitements insecticides (Oerke, 2006). Plus de 200 espèces ont été identifiées, mais seules quelques-unes sont réellement très nuisibles (Renou et Brévault, 2016). Qu'il s'agisse de destruction partielle ou totale d'organes, de ponction de sève, d'injection de salive toxique ou de transmission de germes, ces attaques affectent, 
directement ou indirectement, le rendement et la qualité de la production. Les insectes les plus nuisibles sont les chenilles de la capsule (Helicoverpa armigera Hübner, Diparopsis watersi Rothschild et Earias sp, dites chenilles « exocarpiques »), qui provoquent des chutes d'organes fructifères, boutons floraux et jeunes capsules, et détériorent la fibre des capsules plus âgées. Dans les zones les plus au sud, les chenilles dites «endocarpiques», Thaumatotibia leucotreta (Meyrick), et dans une moindre mesure, Pectinophora gossypiella (Saunders), sont les principaux ravageurs des capsules. Le cortège d'insectes piqueurs suceurs vient immédiatement après, en termes d'importance économique. Il comprend des pucerons (Aphis gossypii Glover), aleurodes (Bemisia tabaci Gennadius), jassides (Empoasca sp), et punaises (Dysdercus fasciatus Signoret, D. völkeri Schmidt et D. superstitiosus Fabricius), dont les mirides (Campylomma sp, C. nicolasi Puton \& Reuter, C. unicolor Poppius, Creontiade pallidus Rambur, Euristylus oldi Poppius, Helopelthis shoutedeni Reuter, Megacoelum apicale Reuter, M. scutellare Poppius, Stenocapsus leucochilus Reuter et Taylorilygus vosseleri Poppius), qui peuvent réduire significativement la production par affaiblissement de la vigueur des plants, abscission d'organes fructifères, transmission de maladies, ou développement d'agents responsables de pourritures détruisant le contenu des capsules, et dépôts de miellats sur la fibre. Les chenilles phyllophages (surtout Haritalodes derogata Fabricius mais aussi Spodoptera littoralis Boisduval et Anomis flava Fabricius) sont fréquemment observées en culture cotonnière. À l'échelle d'une parcelle où leur présence est souvent liée à des défaillances dans la protection phytosanitaire, l'incidence de ces ravageurs est en général faible car les infestations sont souvent très localisées.

Le recours aux pesticides, comme principal moyen de lutte, a montré certaines limites comme des risques pour la santé humaine, le développement de résistances des cibles, mais également un effet délétère sur les écosystèmes, avec une altération des services de régulation naturelle (Barzman et al., 2015). S'ajoutent à cela une réduction de la gamme de molécules disponibles et l'émergence de nouvelles problématiques liées au changement de statut de ravageurs secondaires (e.g., jassides), ou à l'invasion de ravageurs exotiques, comme celle très récente de la noctuelle Spodoptera frugiperda (Goergen et al., 2016: Brévault et al., 2018). Enfin, la simplification des paysages agricoles contribue à l'érosion de la diversité cultivée et à la fragmentation, voire à la disparition des habitats non cultivés, supports de la biodiversité fonctionnelle. Cette érosion de la biodiversité et la perte de fonction de régulation qui l'accompagne accroissent la sensibilité des écosystèmes cultivés aux ravageurs.

Les filières cotonnières d'Afrique de l'Ouest se sont engagées depuis longtemps, à des degrés divers, dans des démarches de gestion «intégrée » des ravageurs de la culture, comprenant certaines mesures prophylactiques (variétés résistantes, semis précoce, destruction des résidus de culture, etc.) et la prise en compte du faciès parasitaire (chenilles endovs. exocarpiques), pour raisonner l'utilisation des insecticides (4-7 traitements sur le cycle du cotonnier) et limiter en amont les risques économiques, sanitaires et environnementaux. Toutefois, les programmes de protection phytosanitaires proposés aux producteurs restent basés le plus souvent sur des traitements systématiques sur calendrier (en général un traitement tous les 14 jours à partir de $30-45$ jours après levée) et l'utilisation d'insecticides à large spectre d'action qui n'épargnent pas les auxiliaires des cultures. Un changement de paradigme dans les pratiques reste donc à opérer pour construire des systèmes de production plus économes en pesticides et plus résilients, favorisant les services écosystémiques de régulation des bioagresseurs, du champ cultivé au territoire.

\section{Gestion intégrée des ravageurs}

Selon la FAO (2014), la gestion «intégrée» des bioagresseurs des cultures (IPM-Integrated Pest Management) est une approche qui combine différentes stratégies et pratiques pour maintenir les cultures en bonne santé et minimiser l'utilisation de pesticides. Elle s'appuie en premier lieu sur la surveillance du niveau des populations de bioagresseurs ou des dégâts associés, permettant le raisonnement des traitements phytosanitaires et l'utilisation privilégiée de matières actives sélectives et à faible empreinte écologique, par opposition au suivi d'un calendrier de traitement et à l'emploi d'insecticides à large spectre. Le concept a été développé en même temps que celui de seuil économique d'intervention, seuil fixant la densité de population de bioagresseurs à partir de laquelle une décision d'intervention doit être prise pour empêcher la population de ravageurs de provoquer des dégâts dont l'incidence économique serait supérieure au coût d'une intervention (Stern et al., 1959). Cette gestion «intégrée» s'appuie aussi sur le recours à une combinaison de méthodes alternatives de contrôle ou de lutte (Fig. 1) qui concourent à limiter l'incidence des bioagresseurs.

\subsection{Contrôle cultural}

En Afrique de l'Ouest, la réalisation de semis précoces est recommandée pour éviter les fortes infestations de chenilles de la capsule en fin de campagne. La mise en place de variétés à cycle court, de fortes densités de semis, ou l'utilisation de régulateurs de croissance, participent de la même stratégie d'évitement des infestations de chenilles de la capsule en fin de campagne (Javaid, 1995). Une fertilisation équilibrée contribue à réduire l'incidence des ravageurs, parce qu'elle autorise la plante à compenser les premières pertes de récolte et qu'elle limite l'exubérance végétative (Vaissayre et al., 2006). L'arrachage des repousses de cotonniers est une pratique efficace, ces repousses servant au maintien de certains ravageurs, en particulier les jassides et les pucerons. L'écimage des cotonniers en fin de cycle est une technique qui permet de limiter les dégâts liés au collage de la fibre, en privant les piqueurs-suceurs d'un support essentiel à leur multiplication (Deguine et al., 2000). L'utilisation de plantes de biocontrôle sous la forme de couverture végétale du sol, de cultures intercalaires ou en rotation, reste insuffisamment explorée en Afrique de l'Ouest. En Australie, des cultures comme le sorgho ou la luzerne sont considérées comme favorables pour le maintien d'ennemis naturels des ravageurs (Mensah et Singleton, 2004). La luzerne (Medicago sativa), en association avec le coton, peut également jouer un rôle de piège pour les mirides (Mensah et Khan, 1997). En Chine, le coton est souvent semé au printemps entre les lignes de blé d'hiver, ce 


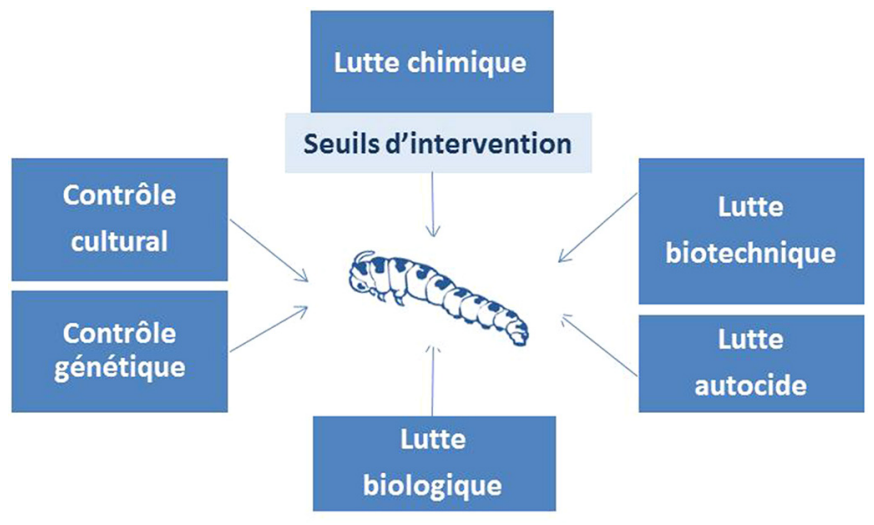

Fig. 1. Les différentes méthodes de contrôle ou de lutte développées contre les bioagresseurs. Ces méthodes alternatives peuvent être mobilisées pour agir sur la population initiale de bioagresseurs, pour éviter la concordance entre la phase de colonisation par le bioagresseur et la période de sensibilité de la culture (évitement), ou pour minimiser les dégâts lorsque la culture et le bioagresseur se trouvent en contact (atténuation). Enfin, les solutions de rattrapage, comme les traitements insecticides, permettent de limiter les dégâts lorsque les leviers mis en œuvre auparavant n'ont pas été efficaces (Attoumani-Ronceux et al., 2010).

Fig. 1. The different control methods developed against pests. These alternative methods can be mobilized to act on the initial pest population, to avoid the coincidence between crop colonization and crop susceptibility (avoidance), or to minimize damage when pest and crop get in contact (attenuation). Finally, catch-up solutions such as insecticide sprayings can limit damage when the levers implemented previously have not been effective (Attoumani-Ronceux et al., 2010).

qui limite l'incidence des pucerons en début de saison. La culture de la luzerne autour des champs de coton constitue une pouponnière pour les coccinelles, les chrysopes et d'autres arthropodes bénéfiques (Lin et al., 2003). Au Brésil, l'association du coton avec Foeniculum vulgare, qui abrite le puceron Hyadaphis foniculi, permet d'augmenter l'impact des prédateurs (Ramalho et al., 2012).

\subsection{Contrôle génétique}

Les caractères de résistance ou de tolérance aux ravageurs les plus utilisés dans la zone cotonnière d'Afrique de l'Ouest sont la résistance à la bactériose et à la mosaïque, et la pilosité foliaire, qui constitue une barrière physique à la prise de nourriture et surtout à l'oviposition des jassides (Parnell et al., 1949). Les potentialités qu'offre la diversité génétique du cotonnier pour la résistance aux ravageurs restent cependant insuffisamment exploitées dans les programmes de sélection variétale. L'utilisation de coton transgénique produisant des toxines provenant de la bactérie du sol Bacillus thuringiensis (Bt) permet de protéger la culture contre certains lépidoptères phytophages (Showalter et al., 2009). Les bénéfices potentiels associés aux cotonniers transgéniques insecticides sont une diminution du recours aux traitements insecticides contre les lépidoptères ravageurs, la conservation des ennemis naturels, un contrôle des ravageurs ciblés à une échelle régionale, et des rendements accrus ou plus stables (Carrière et al., 2003 ; Qaim et Zilberman, 2003; Bennett et al., 2006; Cattaneo et al., 2006; Showalter et al., 2009). Leur diffusion pose cependant la question des risques agronomiques et écologiques, qui incluent les flux de gènes, le développement de résistances chez les insectes, et des effets non intentionnels sur des espèces non ciblées, ou la résurgence de ravageurs secondaires (Luo et al., 2014). Des cotonniers transgéniques de deuxième génération, incluant notamment des ARN interférents (gene silencing), sont en cours d'homologation (Tabashnik et Carrière, 2017).

\subsection{Seuils d'intervention}

Deux grands types de programmes de protection sur seuil pour le contrôle des chenilles de la capsule sont pratiqués en culture cotonnière en Afrique de l'Ouest : un programme dit de «Lutte étagée ciblée» (LEC), qui comprend des traitements d'assurance à demi-dose (dits «filets de protection») et un programme appliquant strictement le principe des seuils. Les seuils d'intervention ont été définis de manière empirique et varient selon les pays (Silvie et al., 2013). Au Mali, le programme LEC est considéré comme une étape de transition vers l'emploi des seuils au sens strict. En 2010, 66\% des surfaces (280 $000 \mathrm{ha}$ ) étaient conduits de manière conventionnelle, $24 \%$ selon le programme LEC, et $10 \%$ sous le régime de seuils stricto sensu. Ce programme LEC a permis une économie d'insecticides de $50 \%$ par rapport aux applications conventionnelles (Michel et al., 2000). Au Cameroun, une méthode d'échantillonnage séquentiel a été mise au point afin de faciliter les observations au champ et la prise de décision (Brévault et al., 2009). Une planchette spécifique a été diffusée auprès des producteurs intéressés. Ce programme LOIC (Lutte sur observation individuelle des chenilles), qui concernait près de $20 \%$ des superficies en 2017 , ne procure pas toujours des économies d'insecticides car son principal atout réside dans un meilleur positionnement des traitements insecticides par rapport à la dynamique des infestations en chenilles de la capsule. Une évolution vers la définition de seuils dynamiques, tenant compte des processus d'élaboration de la production associés aux capacités de compensation de la plante, du potentiel de rendement, ou de la présence d'ennemis naturels, reste à établir pour rationaliser encore davantage l'utilisation d'insecticides (Renou et Brévault, 2016).

\subsection{Lutte biologique}

L'introduction ou les lâchers à grande échelle d'ennemis naturels pour limiter les populations de ravageurs du cotonnier, comme réalisés en Égypte (Baraka et al., 2008), ne sont pas encore d'actualité en Afrique de l'Ouest. Le potentiel d'impact des ennemis naturels est souvent difficile à démontrer, en raison de la gamme des ravageurs à contrôler, du caractère annuel de la culture, et de l'importance accordée aux traitements insecticides en début de saison, souvent avec des insecticides à large spectre (Deguine et al., 2008). En Australie, l'accent est mis sur la conservation des ennemis naturels indigènes (Wilson et al., 2004), avec la supplémentation alimentaire et l'application d'insecticides sélectifs (Mensah et Singleton, 2004) pour maintenir en place les populations de prédateurs. 
Les méthodes de lutte biologique reposant sur l'emploi de préparations à base de virus (baculovirus) ou de bactéries ( $B$. thuringiensis) n'ont connu une diffusion que sur de faibles superficies. En culture biologique au Bénin, leur efficacité a pu atteindre celle d'une protection par des insecticides chimiques grâce à l'adjonction de phagostimulants (Mensah et al., 2012). En Chine, une lutte biologique avec des parasitoïdes (Trichogramma spp. et Microplitis mediator), acariens prédateurs (Amblyseius cucumeris, et biopesticides à base de bactéries (Bacillus thuringiensis) et virus (polyhédrose nucléaire) a été développée pour lutter contre $H$. armigera (Luo et al., 2014). En Australie, une formulation virale à base de polyhédrose nucléaire est commercialisée pour le contrôle d'Helicoverpa spp. (Gregg et al., 2010). En Californie, les tentatives de déclenchement d'épizooties chez $A$. gossypii ou $B$. tabaci par pulvérisation de champignons entomopathogènes se sont soldées par des échecs, la propagation du pathogène étant sous la dépendance de facteurs abiotiques difficilement contrôlables (Steinkraus et al., 2002). Le déploiement de ces biopesticides reste limité par leurs conditions d'utilisation (spectre d'action étroit, efficacité sur les jeunes stades des ravageurs, stockage à température contrôlée, etc.) et leur coût (Renou et Brévault, 2016).

\subsection{Lutte biotechnique}

Parmi les plantes les plus utilisées pour fabriquer des pesticides d'origine naturelle figure le neem, Azadirachta indica, dont les préparations à base de graines ou de feuilles perturbent le comportement des ravageurs (Gahukar, 2000). Elles peuvent être employées seules ou associées à d'autres agents biologiques (Togbe et al., 2015). En Australie, un produit à base d'huile de Clitoria ternatea (Fabaceae) est utilisé pour son effet dissuasif sur l'oviposition et l'alimentation des larves d'Helicoverpa spp. (Mensah et al., 2015). L'utilisation de médiateurs chimiques ou substances sémiochimiques régissant les relations plantes-insectes constitue une voie en plein essor. Les médiateurs chimiques sont des molécules que les insectes détectent via leurs systèmes olfactifs et gustatifs et qui affectent leur comportement ou leur reproduction. Certains sont utilisés avec succès en protection des cultures pour la surveillance des populations de ravageurs (pièges à base de phéromones ou d'appâts alimentaires) ou comme moyen de lutte (piégeage de masse, lutte attracticide, confusion sexuelle, ou encore technique du push-pull). La stratégie push-pull repose sur la manipulation du comportement des ravageurs via l'introduction de plantes répulsives en association avec la culture principale et de plantes attractives en bordure de la culture (Cook et al., 2007). La confusion sexuelle a été utilisée avec succès contre $P$. gossypiella aux États-Unis, en Israël et en Égypte, mais reste peu pratiquée en raison du nombre d'espèces de ravageurs à contrôler et des difficultés de mise en œuvre de cette technologie. En Australie, des attractifs à base de composés volatiles de plantes et de stimulants alimentaires mélangés à un insecticide ont été développés pour attirer et tuer l'adulte d'Helicoverpa spp. sur les bandes traitées de la culture avant qu'il ne puisse pondre ses œufs (Gregg et al., 2004).

Des substances allélochimiques sont également impliquées dans la réaction induite de défense des plantes, consécutive à une attaque de ravageur, et qui peuvent affecter le comportement, la croissance ou les ravageurs (War et al., 2012) : dissuasion des femelles en quête d'un site de ponte ou attraction des ennemis naturels via l'émission de composés organiques volatiles (HIPV, herbivore-induced plant volatiles), érection de barrières physiques, production de composés toxiques ou répulsifs (protéines de défense, métabolites secondaires comme le gossypol, trichomes), production de ressources pour les ennemis naturels (par exemple, nectaires et nectar extrafloral), ou encore induction des plantes voisines qui vont-elles-mêmes se mettre à produire des molécules de défense (Llandres et al., 2018). Des expérimentations conduites au Mali depuis plusieurs années ont montré que l'écimage des cotonniers permet de réduire significativement l'incidence de plusieurs insectes ravageurs, en particulier les chenilles de la capsule (Renou et al., 2011). Ces effets s'étendent aux plants non écimés voisins de plants écimés. Des stratégies de protection intégrant l'écimage de cotonniers sont en cours de diffusion (Téréta et al., 2016). La compréhension des mécanismes de défense induite de la plante pourrait offrir de nouvelles opportunités pour réduire l'utilisation de pesticides dans les programmes de protection des cultures.

\subsection{Lutte autocide}

La lutte autocide ou technique de l'insecte stérile (TIS) est une méthode de contrôle impliquant l'élevage en masse et la stérilisation d'individus mâles du ravageur ciblé, puis leur lâcher dans une zone prédéfinie, où leur accouplement avec des femelles sauvages ne donnera pas de descendance, entraînant ainsi le déclin de la population de ravageurs. Un programme d'éradication initié en 2002 et utilisant une combinaison de méthodes (confusion sexuelle, applications locales d'insecticides, coton $\mathrm{Bt}$-génétiquement modifié, et lâchers de mâles stériles) a éradiqué le ver rose de la capsule, Pectinophora gossypiella (Lepidoptera, Gelechiidae), de certaines régions du sud-ouest des Etats-Unis et du nord du Mexique. La menace persistante de ré-invasion impose cependant le maintien de la surveillance (Byers et Naranjo, 2014). De par son coût et la logistique inhérente à sa mise en place, cette technique reste difficilement applicable en culture cotonnière lorsque la gamme de ravageurs d'intérêt économique est importante.

\section{Gestion agroécologique des ravageurs}

Si la gestion «intégrée» des bioagresseurs des cultures a permis une réduction de l'utilisation des pesticides et des impacts environnementaux associés, il n'en reste pas moins qu'elle est souvent utilisée comme une boîte à outils, de façon réactive, individuelle, ciblée sur la parcelle ou le ravageur (Brévault et Bouyer, 2014). Ainsi, la gestion intégrée des bioagresseurs n'est pas une approche agroécologique si elle consiste à raisonner les traitements chimiques sans réaliser les changements nécessaires de pratiques agricoles ou de système de production. L'activation des services de régulation écologique des bioagresseurs constitue une voie à explorer pour réduire la dépendance des agriculteurs aux pesticides et inventer des modèles de gestion agroécologique des systèmes de protection des cultures. Elle passe par une connaissance approfondie du «système de vie» des populations de 
ravageurs et de leurs ennemis naturels, de leurs interactions au sein des agroécosystèmes (réseaux trophiques) et la prise en compte de l'effet des pratiques agricoles et de l'ensemble des habitats cultivés et non cultivés dans la dynamique de leurs populations. Elle passe aussi par un renforcement des systèmes d'apprentissage des producteurs, comme par exemple dans le cadre de champs-écoles (Prudent et al., 2006), basés sur une approche participative du processus d'innovation.

\subsection{Favoriser les processus de régulation}

La régulation des bioagresseurs est un des services écosystémiques fournis par la biodiversité (Crowder et Jabbour, 2014). Cette régulation s'exerce via les ressources utilisées par les bioagresseurs dans leur habitat (régulation bottom-up), et via les ennemis naturels (régulation top-down) tels que prédateurs, parasitoïdes et pathogènes (Fig. 2).

Au niveau du champ cultivé, les produits de biocontrôle comme les macroorganismes (prédateurs et parasitoïdes), microorganismes entomopathogènes (champignons, bactéries, virus), médiateurs chimiques et autres attractifs/répulsifs naturels, ou substances naturelles (extraits de plantes, éliciteurs naturels), mais aussi les pratiques agricoles comprenant le choix variétal et la diversification du peuplement végétal (plantes de services, bandes fleuries, couverts végétaux, rotations) (Ratnadass et al., 2012), constituent des leviers d'action mobilisables par l'agriculteur pour opposer des barrières physiques ou chimiques aux bioagresseurs, stimuler les mécanismes de défense ou de compensation de la culture, ou encore favoriser le recrutement d'ennemis naturels. Il convient toutefois de prendre en compte le contexte paysager pour identifier les leviers d'action adaptés au système de culture, car les processus de dispersion des ravageurs et de leurs ennemis naturels sont fonction des habitats adjacents et des caractéristiques du paysage environnant (Schellhorn et al., 2015). En outre, on observe une variabilité importante de l'effet des pratiques sur la régulation naturelle, du fait d'une interaction forte avec l'environnement paysager (présence d'habitats semi-naturels, traitements insecticides sur les parcelles alentours, etc.) de la parcelle (Tscharntke et al., 2012).

Au niveau du paysage, l'arrangement spatial des cultures peut retarder leur colonisation par les bioagresseurs, tandis que les éléments de végétation semi-naturelle (arbres, haies, parcours, forêts, etc.) peuvent constituer des habitats qui fournissent des ressources (proies ou hôtes alternatifs, pollen, nectar, refuges) pour différents ennemis naturels et donc servir de support à la régulation écologique des insectes ravageurs des cultures (Rusch et al., 2010). De manière générale, la régulation écologique des bioagresseurs augmente avec la complexité du paysage (Veres et al., 2013). L'aménagement ou la conservation de ces habitats seminaturels dans le paysage sont donc fondamentaux pour la mise en œuvre des stratégies de lutte biologique par conservation. La notion de service écosystémique aborde ici la question des alternatives aux pesticides non pas par l'agronomie comme c'est le cas pour la lutte intégrée, l'agroécologie ou l'agriculture biologique, mais par une analyse du fonctionnement des écosystèmes en lien avec la durabilité des systèmes de production agricole.

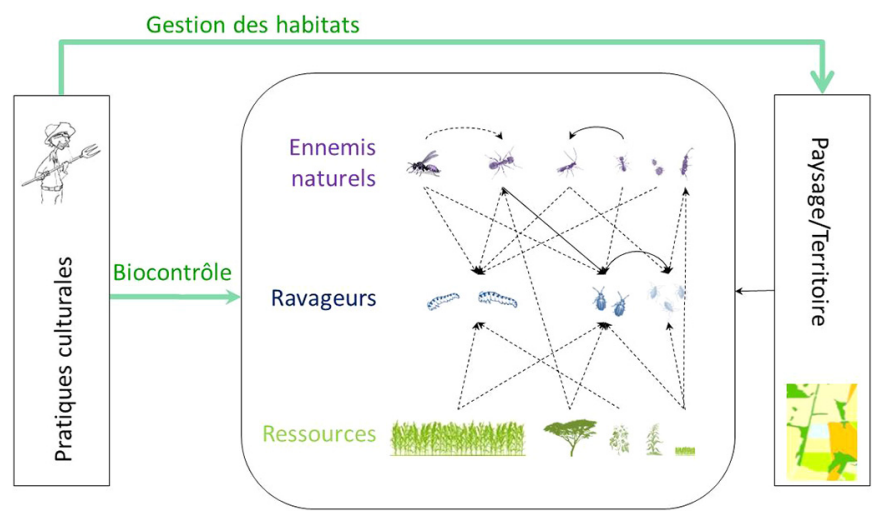

Fig. 2. Leviers de régulation des populations d'insectes ravageurs des cultures (d'après Brévault et Clouvel, 2019).

Fig. 2. Regulation levers of insect pest populations (adapted from Brévault and Clouvel, 2019).

\subsection{Composer les actions et les échelles}

Le paysage, en interaction constante avec les processus écologiques qui s'y déroulent, est la résultante de la dynamique du milieu et de la société qui s'y est développée (Burel et Baudry, 1999). De par sa structure et sa composition (mosaïque d'habitats), il influence la dispersion et la dynamique des populations des ravageurs et de leurs ennemis naturels. Il est sans doute le niveau d'analyse approprié pour une approche holistique de l'action, considérant les pratiques individuelles et collectives en matière de lutte contre les bioagresseurs, leur efficience et leurs externalités, dans la perspective d'une gestion durable de la production. La démarche que nous proposons pour une gestion agroécologique des bioagresseurs consiste à composer :

- l'action individuelle sur le milieu au travers des pratiques et des innovations techniques compatibles avec les processus de régulation écologique;

- l'action collective sur un territoire au travers d'une gestion coordonnée des ravageurs (gestion territoriale des ravageurs) ou d'aménagement des habitats pour favoriser les processus de régulation par les ennemis naturels (gestion paysagère des ravageurs et de leurs ennemis naturels); la gestion paysagère intègre, en plus de la dimension territoriale, l'hétérogénéité du paysage.

Cette démarche implique un changement fondamental des contours de l'action, d'une approche individuelle à l'échelle de la parcelle, à une organisation territorialisée de stratégies de lutte et de gestion des services écosystémiques incluant des dimensions écologiques, économiques et sociales (Cong et al., 2014; Brévault et al., 2014; Brévault et Clouvel, 2019).

Le lâcher à grande échelle de mâles stériles pendant quatre ans en Arizona a permis de limiter l'évolution de la résistance $\mathrm{du}$ ver rose de la capsule au coton $\mathrm{Bt}$, et en association avec d'autres méthodes (coton $\mathrm{Bt}$, confusion sexuelle) a réduit l'abondance du ravageur de plus de $99 \%$ (Tabashnik et al., 2010), avant de le déclarer officiellement éradiqué (Mace, 2018). En Australie, des stratégies volontaristes ont été mises au point pour ralentir le développement de la résistance aux 
insecticides, incluant la rotation des matières actives et des traitements sur seuil. Malgré l'adoption de ces pratiques, la résistance aux insecticides a continué à se développer jusqu'à ce que l'introduction du coton Bt et le plan associé de gestion de la résistance fasse passer les exploitations d'une approche individuelle à une approche coordonnée à l'échelle du paysage, tenant compte des plantes-hôtes alternatives du ravageur avant et après la culture du coton (Downes et al., 2017). En matière de gestion paysagère des ravageurs, des travaux de Carrière et al. (2006) ont montré que des arrangements des parcelles de luzerne et des jachères (sources) par rapport aux parcelles de coton, dans le temps ou dans l'espace, réduisaient significativement les dégâts de la punaise Lygus hesperus (Heteroptera, Miridae) sur le coton. Dans la vallée de San Joaquin en Californie, la gestion du ravageur par la conservation de bandes de luzerne non récoltées a permis une baisse significative de l'utilisation d'insecticide, avec moins d'une application par saison (Goodell, 2009). En Chine, Liu et al. (2016) ont montré que la diversité des paysages augmentait le parasitisme des œufs d'H. armigera par Trichogramma chilonis en culture de coton.

\subsection{Intégrer et former les acteurs}

Des études sur l'impact du coton Bt ont montré que sa culture intensive dans le nord de la Chine permettait de contrôler non seulement $H$. armigera, et $P$. gossypiella, mais réduisait également sa présence sur d'autres cultures hôtes (effet de halo) ou sur le coton non-Bt, respectivement, diminuant ainsi l'utilisation d'insecticides dans la région $(\mathrm{Wu}$ et al., 2008; Wan et al., 2012). Dans les bassins de production où les agriculteurs sont confrontés à des ravageurs communs, il peut être intéressant pour eux de coordonner leur action à l'échelle régionale. On peut citer ici, comme exemple de coordination à l'échelle d'un territoire, la mise en place d'un programme de gestion de la résistance d'H. armigera aux pyréthrinoïdes en culture cotonnière en Afrique de l'Ouest (Martin et al., 2005). Si cette initiative a été un succès pour la maîtrise de la résistance et le partage des connaissances, les producteurs maraîchers, eux aussi impactés par la résistance de ce ravageur dans les territoires concernés, n'ont pas participé à sa mise en œuvre mais ont profité de ses retombées. L'expérience de cette mise en place de gestion concertée des ravageurs a permis d'approcher les défis d'organisation que pose une approche collective et territoriale, et la nécessité d'intégrer le maximum d'acteurs avant d'entreprendre l'action collective considérée.

Les travaux de recherche sur les systèmes agricoles et la complexité des processus d'adoption de méthodes agroécologiques doivent conduire à interagir en amont du processus d'innovation avec les acteurs des territoires concernés. Il faut que les acteurs se représentent les bénéfices, issus du paysage ou des effets de ce paysage sur les ennemis naturels, pouvant être obtenus en agissant sur celui-ci. Ensuite, il faut qu'une partie des acteurs se représente cette possibilité comme dépendante des autres acteurs. Les représentations des acteurs sont à la source de leurs actions. Comme montré par des enquêtes auprès d'arboriculteurs du sud de la France, le paysage n'est pas toujours considéré comme une ressource pour la lutte biologique contre les ravageurs, mais plutôt comme une source de ravageurs (Salliou et Barnaud, 2017). Le succès d'une telle approche repose donc sur la perception par les acteurs d'intérêts communs à résoudre un problème partagé. Une coopération dans la gestion des ressources naturelles à l'échelle du territoire (agriculteurs, organisations paysannes, décideurs politiques, etc.), soutenue par des sessions de formation, et la mise en place de règles de mise en œuvre des programmes de lutte ou d'aménagement du paysage favorisant les services écosystémiques, constituent le pilier de l'action.

\section{Conclusion}

La mise au point et la diffusion de solutions alternatives aux pesticides constitue un enjeu stratégique pour une production cotonnière durable en Afrique de l'Ouest. Avec la mise en place de méthodes prophylactiques, l'introduction de biopesticides et de nouveaux programmes de traitements sur seuils comme version évoluée de la lutte étagée ciblée, certains pays se sont déjà engagés dans cette voie. Ainsi, malgré son image de culture dépendante des pesticides (Djagni et Fok, 2019), la culture cotonnière continue à constituer un cas d'école pour la conception de nouveaux systèmes de protection des plantes cultivées (Ferron et al., 2006). Le changement des pratiques, au bénéfice de la mobilisation des services de régulation écologique des bioagresseurs, constitue encore une voie à explorer pour mettre au point des modèles d'intensification écologique de la production agricole. Ceux-ci doivent permettre d'accroître la productivité des systèmes agricoles (produire plus) et de diminuer la dépendance des producteurs vis-à-vis des pesticides (produire mieux). Les leviers permettant de favoriser les processus de régulation naturelle des bioagresseurs sont multiples et redevables d'actions aux différentes échelles, du champ cultivé au territoire, mais nécessitent encore d'approfondir notre connaissance du système de vie des bioagresseurs et de leurs ennemis naturels, pour construire des règles de décision tenant compte du contexte paysager. Ils nécessitent aussi l'accompagnement de la recherche pour la diffusion des innovations au sein des filières et leur adoption par les producteurs de coton. À terme, il s'agit de construire une organisation territorialisée d'actions individuelles et collectives de gestion des ravageurs et des services écosystémiques, incluant des dimensions écologiques, économiques et sociales.

Remerciements. Ce travail a reçu le soutien du Cirad et du dispositif d'enseignement et de recherche en partenariat Divecosys (https://www.divecosys.org/presentation).

\section{Références}

Attoumani-Ronceux A, Aubertot JN, Guichard L, Jouy L, Mischler P, Omon B, et al. 2010. Guide pratique pour la conception de systèmes de culture plus économes en produits phytosanitaires. Application aux systèmes de polyculture. Ministères chargés de l'agriculture et de l'environnement, RMT SdCi.

Baraka MR, Bahira ME, Alia MAE, Abd ER, Imam AI. 2008. Evaluation of the number of releases of the egg parasitoid, Trichogramma evanescens West, in suppressing the spiny bollworm, Earias insulana (Boisd.) infestation in El-Farafra 
cotton fields, New Valley Governorate, Egypt. Egyptian Journal of Biological Pest Control 18: 271-275.

Barzman M, Bàrberi P, Birch ANE, Boonekamp P, DachbrodtSaaydeh S, Graf B, et al. 2015. Eight principles of integrated pest management. Agronomy for Sustainable Development 35: 1199-1215.

Bennett R, Morse S, Ismael Y. 2006. The economic impact of genetically modified cotton on South African smallholders: Yield, profit and health effects. The Journal of Development Studies 42: 662-677.

Brévault T, Bouyer J. 2014. From integrated to system-wide pest management: Challenges for sustainable agriculture. Outlooks on Pest Management 25: 212-213.

Brévault T, Clouvel P. 2019. Pest management: Reconciling farming practices and natural regulations. Crop Protection 115: 1-6.

Brévault T, Renou A, Vayssières JF, Amadji GL, Assogba-Komlan F, Diallo MD, et al. 2014. DIVECOSYS: Bringing together researchers to design ecologically-based pest management for small-scale farming systems in West Africa. Crop Prot 66: 53-60.

Brévault T, Ndiaye A, Badiane D, Bal A, Sembène M, Silvie P, et al. 2018. First records of the fall armyworm, Spodoptera frugiperda (Lepidoptera: Noctuidae), in Senegal. Entomologia Generalis 37: 129-142.

Burel F, Baudry J. 1999. Écologie du paysage: Concepts, méthodes et applications. Paris : Lavoisier.

Brévault T, Couston L, Bertrand A, Thézé M, Nibouche S, Vaissayre M. 2009. Sequential pegboard to support small farmers in cotton pest control decision-making in Cameroon. Crop Protection 28: 968-973.

Byers JA, Naranjo SE. 2014. Detection and monitoring of pink bollworm moths and invasive insects using pheromone traps and encounter rate models. Journal of Applied Ecology 51: 1041-1049.

Carrière Y, Ellers-Kirk C, Sisterson M, Antilla L, Whitlow M, Dennehy TJ, et al. 2003. Long-term regional suppression of pink bollworm by Bacillus thuringiensis cotton. Proceedings of the National Academy of Sciences 100: 1519-1523.

Carrière Y, Ellsworth PC, Dutilleul P, Ellers-Kirk C, Barkley V, Antilla L. 2006. A GIS-based approach for areawide pest management: The scales of Lygus hesperus movements to cotton from alfalfa, weeds, and cotton. Entomologia Experimentalis et Applicata 118: 203-210.

Cattaneo MG, Yafuso C, Schmidt C, Huang CY, Rahman M, Olson C, et al. 2006. Farm-scale evaluation of the impacts of transgenic cotton on biodiversity, pesticide use, and yield. Proceedings of the National Academy of Sciences 103: 7571-7576.

Cong R-G, Smith HG, Olsson O, Brady M. 2014. Managing ecosystem services for agriculture: Will landscape-scale management pay? Ecological Economics 99: 53-62.

Cook SM, Khan ZR, Pickett JA. 2007. The use of push-pull strategies in integrated pest management. Annual Review of Entomology 52: 375-400.

Crowder D, Jabbour L. 2014. Relationships between biodiversity and biological control in agroecosystems: Current status and future challenges. Biological Control 75: 8-17.

Deguine JP, Gozé E, Leclant F. 2000. The consequences of late outbreaks of the aphid Aphis gossypii in cotton growing in central Africa: Towards a possible method for the prevention of cotton stickiness. International Journal of Pest Management 46: 85-89.

Deguine JP, Ferron P, Russell D. 2008. Sustainable pest management for cotton production. A review. Agronomy for Sustainable Development 28: 113-137.

Djagni KK, Fok M. 2019. Dangers potentiels de l'utilisation des insecticides dans la culture cotonnière au Togo de 1990 à 2010. Cahiers Agricultures 28. DOI: 10.1051/cagri/2019023.
Downes S, Kriticos D, Parry H, Paull C, Schellhorn N, Zalucki MP, et al. 2017. A perspective on management of Helicoverpa armigera: transgenic $\mathrm{Bt}$ cotton, IPM, and landscapes. Pest Management Science 73: 485-492.

FAO. 2014. Integrated pest management. http://www.fao.org/agricul ture/crops/thematic-sitemap/theme/pests/ipm/en/. Site consulté le 11/10/2017.

Ferron P, Deguine JP, Ekorong J. 2006. Évolution de la protection phytosanitaire du cotonnier: un cas d'école. Cahiers Agricultures 15: 128-134. Disponible sur http://revues.cirad.fr/index.php/ cahiers-agricultures/article/view/30571.

Gahukar RT. 2000. Use of neem products/pesticides in cotton pest management. International Journal of Pest Management 46: 149-160.

Goergen G, Kumar PL, Sankung SB, Togola A, Tamò M. 2016. First report of outbreaks of the fall armyworm Spodoptera frugiperda (JE Smith) (Lepidoptera, Noctuidae), a new alien invasive pest in West and Central Africa. PLoS ONE 11: e0165632.

Goodell PB. 2009. Fifty years of the integrated control concept: The role of landscape ecology in IPM in San Joaquin valley cotton. Pest Management Science 65: 1293-1297.

Gregg PC, Del Socorro AP, Hawes A. 2004. Magnet : An attract-andkill formulation for Helicoverpa armigera and $H$. punctigera (Lepidoptera: Noctuidae) based on plant volatiles. In: The 2004 ESA Annual Meeting and Exhibition. Available from http://esa. confex.com/esa/2004/techprogram/paper_14005.htm.

Gregg PC, Greive KA, Del Socorro AP, Hawes AJ. 2010. Research to realisation: The challenging path for novel pest management products in Australia. Austral Entomology 49: 1-9.

Javaid I. 1995. Cultural control practices in cotton pest management in tropical Africa. Journal of Sustainable Agriculture 5: 171-185.

Lin R, Liang H, Zhang R, Tian C, Ma Y. 2003. Impact of alfalfa/cotton intercropping and management on some aphid predators in China. Journal of Applied Entomology 127: 33-36.

Liu B, Yang L, Yang F, Wang Q, Yang Y, Lu Y, et al. 2016. Landscape diversity enhances parasitism of cotton bollworm (Helicoverpa armigera) eggs by Trichogramma chilonis in cotton. Biological Control 93: 15-23.

Llandres AL, Almohamad R, Brévault T, Renou A, Téréta I, Jean J, et al. 2018. Plant training for induced defense against insect pests: A promising tool for integrated pest management in cotton. Pest Management Science 74: 2004-2012.

Luo S, Naranjo SE, Wu K. 2014. Biological control of cotton pests in China. Biological Control 68: 6-14.

Mace M. 2018. How Arizona scientists and farmers banished the pink bollworm from the Southwest. Arizona Daily Star. Consulté le 18/ $01 / 2019$.

Martin T, Ochou Ochou G, Djihinto A, Traore D, Togola M, Vassal JM, et al. 2005. Controlling an insecticide resistance bollworm in West Africa. Agriculture, Ecosystems and Environment 107: 409-411.

Mensah R, Leach D, Young A, Watts N, Glennie P. 2015. Development of Clitoria ternatea as a biopesticide for cotton pest management: Assessment of product effect on Helicoverpa spp. and their natural enemies. Entomologia Experimentalis et Applicata 154: 131-145.

Mensah RK, Khan M. 1997. Use of Medicago sativa interplantings/ trap crops in the management of the green mirid, Creontiades dilutus, in commercial cotton in Australia. International Journal of Pest Management 43: 197-202.

Mensah RK, Singleton A. 2004. Development of IPM in cotton in Australia: Establishment and utilization of natural enemies and integration with biological and synthetic insecticides. In: Swanepoel A, ed. Proc. World Cotton Conf.-3, Cotton production for the new millenium, Cape Town, 2003. Pretoria: ARC, Institute for Industrial Crops, pp. 941-951. 
Mensah RK, Vodouhe DS, Sanfillippo D, Assogba G, Monday P. 2012. Increasing organic cotton production in Benin West Africa with a supplementary food spray product to manage pests and beneficial insects. International Journal of Pest Management 58: 53-64.

Michel B, Togola M, Tereta I, Traore N. 2000. La lutte contre les ravageurs du cotonnier au Mali: problématique et évolution récente. Cahiers Agricultures 9: 109-115. Disponible sur http:// revues.cirad.fr/index.php/cahiers-agricultures/article/view/30228.

Oerke EC. 2006. Crop losses to pests. The Journal of Agricultural Science 144: 31-43.

Parnell FR, King HE, Ruston DF. 1949. Insect resistance and hairiness of the cotton plant. Bulletin of Entomological Research 39: 539-575.

Prudent P, Loko S, Vaissayre M. 2006. Les Écoles paysannes au Bénin: une approche participative de la diffusion des messages relatifs à la protection intégrée du cotonnier. Cahiers Agricultures 15: 100-101. Disponible sur http://revues.cirad.fr/index.php/ cahiers-agricultures/article/view/30566/30326.

Qaim M, Zilberman D. 2003. Yield effects of genetically modified crops in developing countries. Science 299: 900-902.

Ramalho FS, Fernandes FS, Nascimento ARB, Nascimento JL, Malaquias JB, Silva CAD. 2012. Assessment of fennel aphids (Hemiptera: Aphididae) and their predators in fennel intercropped with cotton with colored fibers. Journal of Economic Entomology 105: 113-119.

Ratnadass A, Fernandes P, Avelino J, Habib R. 2012. Plant species diversity for sustainable management of crop pests and diseases in agroecosystems: A review. Agronomy for Sustainable Development 32: 273-303.

Renou A, Brévault T. 2016. Ravageurs et maladies du cotonnier, et gestion intégrée des ravageurs. In : Crétenet M, Gourlot J-P, eds. Le cotonnier. Versailles: Ed. Quae, pp. 109-154.

Renou A, Téréta I, Togola M. 2011. Manual topping decreases bollworm infestations in cotton cultivation in Mali. Crop Protection 30: 1370-1375.

Rusch A, Valantin-Morison M, Sarthou JP, Roger-Estrade J. 2010. Biological control of insect pests in agroecosystems: Effects of crop management, farming systems and semi-natural habitats at the landscape scale. A review. Advances in Agronomy 109: 219-259.

Salliou N, Barnaud C. 2017. Landscape and biodiversity as new resources for agro-ecology? Insights from farmers' perspectives. Ecology and Society 22: 16.

Schellhorn NA, Parry HR, Macfadyen S, Wang Y, Zalucki MP. 2015. Connecting scales: Achieving in-field pest control from areawide and landscape ecology studies. Insect Science 22: 35-51.

Showalter AM, Heuberger S, Tabashnik BE, Carrière Y. 2009. A primer for using transgenic insecticidal cotton in developing countries. Journal of Insect Science 9: 22.
Silvie PJ, Renou A, Vodounnon S, Bonni G, Adegnika MO, Héma O, et al. 2013. Threshold-based interventions for cotton pest control in West Africa: What's up 10 years later? Crop Protection 43: 157-165.

Steinkraus DC, Boys GO, Rosenheim JA. 2002. Classical biological control of Aphis gossypii with Neozygites fresenii in California cotton. Biological Control 25: 297-304.

Stern VM, Smith R, Van den Bosch R, Hagen K. 1959. The integration of chemical and biological control of the spotted alfalfa aphid: The integrated control concept. California Agriculture 29: 81-101.

Tabashnik BE, Carrière Y. 2017. Surge in insect resistance to transgenic crops and prospects for sustainability. Nature Biotechnology 35: 926-935.

Tabashnik BE, Sisterson MS, Ellsworth PC, Dennehy TJ, Antilla L, Liesner L, et al. 2010. Suppressing resistance to Bt cotton with sterile insect releases. Nature Biotechnology 28: 1304.

Téréta I, Brévault T, Sissoko F, Goebel R, Renou A. 2016. Cotton topping as a way to reduce farmer's reliance on insecticides in Mali. World Cotton research Cotton Conference. Goiânia, Goiás, Brazil, May 2-6, 2016.

Togbe CE, Haagsma R, Zannou E, Gbehounou, G, Deguenon JM, Vodouhe S, et al. 2015. Field evaluation of the efficacy of neem oil (Azadirachta indica A. Juss) and Beauveria bassiana (Bals.) Vuill. in cotton production. Journal of Applied Entomology 139: 217-228.

Tscharntke T, Tylianakis JM, Rand TA, Didham RK, Fahrig L, Batary P, et al. 2012. Landscape moderation of biodiversity patterns and processes - eight hypotheses. Biological Reviews 87: 661-685.

Vaissayre M, Ochou Ochou G, Hema OS, Togola M. 2006. Quelles stratégies pour une gestion durable des ravageurs du cotonnier en Afrique subsaharienne? Cahiers Agricultures 15: 80-84. Disponible sur http://revues.cirad.fr/index.php/cahiers-agricultures/ article/view/30563.

Veres A, Petit S, Conord C, Lavigne C. 2013. Does landscape composition affect pest abundance and their control by natural enemies? A review. Agriculture, Ecosystems \& Environment 166 : $110-117$.

Wan P, Huang Y, Tabashnik BE, Huang M, Wu K. 2012. The halo effect: Suppression of pink bollworm on non-Bt cotton by Bt cotton in China. PloS ONE 7: e42004.

War AR, Paulraj MG, Ahmad T, Buhroo AA, Hussain B, Ignacimuthu $\mathrm{S}$, et al. 2012. Mechanisms of plant defense against insect herbivores. Plant Signaling \& Behavior 7: 1306-1320.

Wilson LJ, Mensah RK, Fitt GP. 2004. Implementing integrated pest management in Australian cotton. In: Horowitz AR, Ishaaya I, eds. Insect pest management, field and protected crops. Berlin/ Heidelberg: Springer-Verlag, pp. 97-118.

Wu KM, Lu YH, Feng HQ, Jiang YY, Zhao JZ. 2008. Suppression of cotton bollworm in multiple crops in China in areas with Bt toxin-containing cotton. Science 321: 1676-1678.

Citation de l'article : Brévault T, Badiane D, Goebel R, Renou A, Téréta I, Clouvel P. 2019. Repenser la gestion des ravageurs du cotonnier en Afrique de l'Ouest. Cah. Agric. 28: 25. 